ORIGINAL ARTICLE

\title{
Overexpression of ribosomal RNA in prostate cancer is common but not linked to rDNA promoter hypomethylation
}

\author{
M Uemura ${ }^{1}$, Q Zheng ${ }^{1}$, CM Koh ${ }^{1}$, WG Nelson ${ }^{1,2,3,4,5}$, S Yegnasubramanian ${ }^{2,4}$ and \\ AM De Marzo $1,2,3,4,5$
}

${ }^{1}$ Department of Pathology, The Johns Hopkins University School of Medicine, Baltimore, MD, USA; ${ }^{2}$ Department of Oncology, The Johns Hopkins University School of Medicine, Baltimore, MD, USA; ${ }^{3}$ Department of Urology, The Johns Hopkins University School of Medicine, Baltimore, MD, USA; ${ }^{4}$ The Sidney Kimmel Comprehensive Cancer Center at Johns Hopkins, The Johns Hopkins University School of Medicine, Baltimore, MD, USA and ${ }^{5}$ The James Buchanan Brady Urological Institute, The Johns Hopkins University School of Medicine, Baltimore, MD, USA

\begin{abstract}
Alterations in nucleoli, including increased numbers, increased size, altered architecture and increased function are hallmarks of prostate cancer cells. The mechanisms that result in increased nucleolar size, number and function in prostate cancer have not been fully elucidated. The nucleolus is formed around repeats of a transcriptional unit encoding a $45 \mathrm{~S}$ ribosomal RNA (rRNA) precursor that is then processed to yield the mature 18S, 5.8S and 28S RNA species. Although it has been generally accepted that tumor cells overexpress rRNA species, this has not been examined in clinical prostate cancer. We find that indeed levels of the 45S rRNA, 28S, 18S and 5.8S are overexpressed in the majority of human primary prostate cancer specimens as compared with matched benign tissues. One mechanism that can alter nucleolar function and structure in cancer cells is hypomethylation of $\mathrm{CpG}$ dinucleotides of the upstream rDNA promoter region. However, this mechanism has not been examined in prostate cancer. To determine whether rRNA overexpression could be explained by hypomethylation of these CpG sites, we also evaluated the DNA methylation status of the rDNA promoter in prostate cancer cell lines and the clinical specimens. Bisulfite sequencing of genomic DNA revealed two roughly equal populations of loci in cell lines consisting of those that contained densely methylated deoxycytidine residues within CpGs and those that were largely unmethylated. All clinical specimens also contained two populations with no marked changes in methylation of this region in cancer as compared with normal. We recently reported that MYC can regulate rRNA levels in human prostate cancer; here we show that MYC mRNA levels are correlated with $45 \mathrm{~S}, 18 \mathrm{~S}$ and $5.8 \mathrm{~S}$ rRNA levels. Further, as a surrogate for nucleolar size and number, we examined the expression of fibrillarin, which did not correlate with rRNA levels. We conclude that rRNA levels are increased in human prostate cancer, but that hypomethylation of the rDNA promoter does not
\end{abstract}

Correspondence: Professor AM De Marzo, Department of Pathology, Johns Hopkins University School of Medicine, CRB II-144, 1550 Orleans Street, Baltimore, MD 21287, USA.

E-mail: ademarz@jhmi.edu

Received 16 February 2011; revised 14 May 2011; accepted 17 June 2011; published online 8 August 2011 explain this increase, nor does hypomethylation explain alterations in nucleolar number and structure in prostate cancer cells. Rather, rRNA levels and nucleolar size and number relate more closely to MYC overexpression.

Oncogene (2012) 31, 1254-1263; doi:10.1038/onc.2011.319; published online 8 August 2011

Keywords: epigenetics; rDNA; rRNA; DNA methylation; prostate cancer

\section{Introduction}

Changes in nuclear morphology such as enlargement and increased numbers of nucleoli are diagnostic hallmarks of many types of cancer cells, including prostate cancer and its putative precursor lesion, high-grade prostatic intraepithelial neoplasia (Bostwick et al., 1996; Epstein and Yang, 2002; Fischer et al., 2004). The nucleolus is the site of ribosome biogenesis where ribosomal RNA (rRNA) is transcribed, processed, modified and assembled into ribosomal subunits. Regulation of ribosome biogenesis is tightly linked to the control of cellular growth and proliferation. rRNA transcription and ribosome biogenesis are controlled by a number of cellular pathways such as the PI3 kinase/ mTOR, MYC and RAS/ERK pathways (Derenzini et al., 1998; Ruggero and Pandolfi, 2003; Fischer et al., 2004; McStay and Grummt, 2008; Drygin et al., 2010; Grummt, 2010; Grummt and Voit, 2010), and increased rRNA synthesis and ribosome biogenesis are thought to be important molecular alterations that occur very commonly in cancer cells (Ruggero and Pandolfi, 2003; White, 2005; Williamson et al., 2006; Drygin et al., 2010). Therefore, elucidating the mechanisms by which nucleolar enlargement, number and function are deregulated in cancer initiation and progression is likely to be critical to our understanding of abnormal cell proliferation control in prostate cancer.

The nucleolus is formed around tandem repeats of the ribosomal RNA genes (rDNA), each of which contains a promoter region (Figure 1). Human rDNA repeat units comprise $\sim 43 \mathrm{~kb}$ (Gonzalez and Sylvester, 1995). Sequences encoding pre-rRNA (18S, 5.8S and 28S) are 


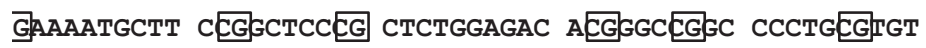
GGCCAGGGCG GCEGGGAGG EGTCCCEGGC CEGGEGCTGC TCCEGCGTT ССTAEGACEG GGACACACGI CCTGGGGTTG ACCAGAGGGC CCEGGGEGT CEGTGTGTGG CTGEGATGGT GGEGTTTTTG GGGACAGGTG TCEGT 35GrCGCCTG GGCEGGCGGE

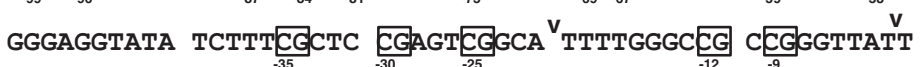

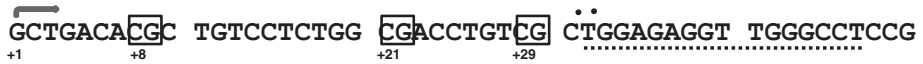

GATGCGCGCG GGGCTCTGGC CTCACGGTGA C

b

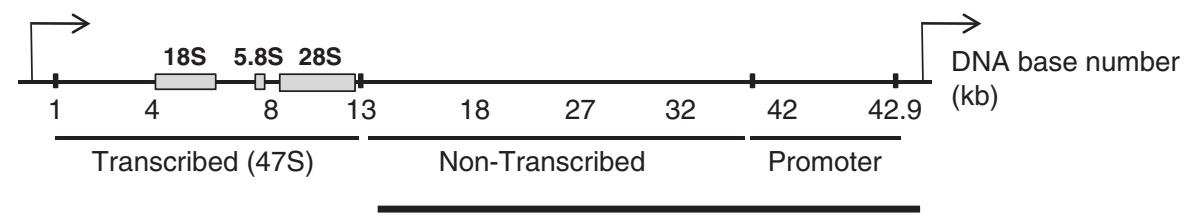

Intrergenic spacer (IGS)

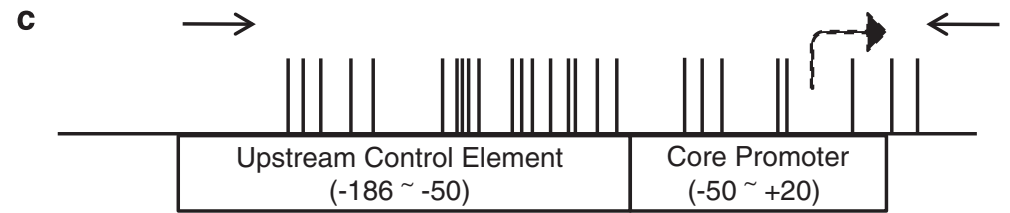

Figure 1 The human rDNA promoter region. (a) Genotyping of rDNA promoter region. The sequence of the rDNA promoter as shown was identical for all prostate cancer tissues and normal tissues, as well as all the cell lines. In this map, primer sequences for genotyping are indicated by the underlined regions and the primer sequences for bisulfite sequencing are indicated as dotted underlines. $\mathrm{CpG}$ dinucleotides are boxed with the location marked relative to the transcription start site (arrow). Discrepancies with the published DNA sequence (U13369) are marked. Nucleotides that show base substitutions as compared with the reference U13369 seqeunce are illustrated with dots above the DNA sequence. Deletions (generally a single base), relative to the reference sequence, are marked by a ' $v$ 'between nucleotides. (b) Schematic representation of a single human rDNA repeat. The approximate positions relative to the transcription start site are indicated (DNA base number in $\mathrm{kb}$ ). (c) Vertical lines indicate locations of CpG dinucleotides on the 45S rRNA promoter relative to the transcription start site, indicated with the dashed curved arrow, with primer pairs used for bisulfite mapping marked by inward pointing arrows.

$\sim 13 \mathrm{~kb}$ and these are separated by intergenic spacers of $\sim 30 \mathrm{~kb}$, which are largely non-transcribed. rDNA repeats are generally found in two major chromatin states. In all species examined, including humans, $\sim 1 / 2$ of the repeat units are present in densely compact 'heterochromatin' that is not accessible to the crosslinking agent psoralen in vivo (McStay and Grummt, 2008). The other $1 / 2$ of the repeats is generally accessible to psoralen and these contain a more open 'euchromatic' chromatin structure. The rDNA promoter consists of a core promoter element adjacent to the transcription start site and a nearby upstream control element (Figures 1b and c) (Haltiner et al., 1986). In human cells, deoxycytidine methylation of the $\sim 25 \mathrm{CpGs}$ residing within the promoter is present in two major patterns. For each repeat unit, either this entire region contains largely unmethylated $\mathrm{CpGs}$ or the entire region contains largely methylated CpGs. In general, $\sim 1 / 2$ of the repeats show each type of configuration (McStay and Grummt, 2008), and the closed psoralen-inacces- sible repeats correspond to those containing densely methylated $\mathrm{CpG}$ dinucleotides in the promoter regions, whereas the psoralen-accessible repeats are largely unmethylated in the promoter regions (McStay and Grummt, 2008). Further, the densely methylated psoralen inaccessible repeats are transcriptionally inactive. And, of the non-methylated repeats, it appears that only a limited number of these units are active at any one time.

Increases in rRNA synthesis can occur by increases in initiation, elongation or processing of previously accessible 'euchromatic' repeats, or by recruitment of previously inactivated heterochromatic repeats to increase the number of active repeats, or by both of these mechanisms (Grummt, 2010). A number of factors activate the expression of rRNA levels by RNA polymerase I in the non-heterochromatic repeats, the most well studied of which is upstream-binding factor. Upstream-binding factor is a high mobility group box DNA-binding protein, which binds to the rDNA promoter and recruits selectivity factor 1 (SL1), a 
TBP-TAFI complex that regulates transcription initiation by RNA polymerase I (Pol I). Upstream-binding factor activity is tightly regulated by association with transcription factors and post-translational modifications such as acetylation and phosphorylation.

In terms of silent heterochromatic regions a number of studies indicate that the nucleolar remodeling complex is required to maintain DNA methylation of the silent repeats by recruiting DNA methyltransferases (Grummt, 2010). In liver cancer, a recent report indicated that the tumor cells showed decreased methylation of the promoter region and that this correlated with increased rRNA transcription (Ghoshal et al., 2004). In prostate cancer, although there are common nucleolar structural abnormalities and increased expression of a number of genes whose products localize to nucleoli (Fischer et al., 2004), (Koh et al., 2010), it has not been determined previously whether rRNA levels are actually increased, nor has it been determined whether cancer-cell-specific epigenetic alterations of the rDNA promoter occur. That altered methylation of the rDNA loci can affect nucleolar structure and function was shown recently as targeted disruption of DNMT1 and/or DMT3b resulted in disrupted nucleolar architecture and complex alterations in rRNA transcription and processing (Espada et al., 2007; Gagnon-Kugler et al., 2009). Therefore, we undertook the current study to examine the hypotheses that rRNA levels are increased in human prostate cancer cells, and that these levels as well as nucleolar structural abnormalities (for example, increased size and number) are related to hypomethylation of the rDNA promoter region.

\section{Results}

To determine whether rRNA levels were elevated in human prostate cancer, we isolated total RNA from a series of tumor and normal (non-neoplastic regions) pairs from fresh frozen tissue samples obtained from radical prostatectomy specimens (Table 1) and performed real time quantitative reverse transcriptase PCR for the $45 \mathrm{~S}$ precursor form as well as the $28 \mathrm{~S}, 18 \mathrm{~S}$ and 5.8S mature rRNA forms (Figure 2). The 45S precursor is very short lived, and is considered a sign of new transcription. Although each of the forms of rRNA measured was higher in tumor samples, as compared with matched normal samples, these increases reached statistical significance only for the $45 \mathrm{~S}$ and $5.8 \mathrm{~S}$ forms. Similar overall results were obtained if we normalized the rRNA species levels to total RNA, as well as to DNA content (data not shown). These results suggest that active transcription of the precursor rRNA transcript, as well as the steady state levels of at least some of the mature rRNA forms, are significantly higher in human prostate cancer samples as compared with matched non-cancerous samples. Table 2 shows that the levels of the various rRNA species correlated with each other in tumor samples. There was no correlation between rRNA levels and Gleason score or pathological stage for any of the species measured (not shown).

There are a number of mechanisms by which rRNA transcription and processing are regulated. One

Table 1 Clinical/pathological characteristics of patient samples

\begin{tabular}{llccccl}
\hline Age & Margins & $\begin{array}{c}\text { Gleason } \\
\text { sum }\end{array}$ & $\begin{array}{c}\text { Gleason } \\
\text { primary }\end{array}$ & $\begin{array}{c}\text { Gleason } \\
\text { secondary }\end{array}$ & $\begin{array}{c}\text { Gleason } \\
\text { tertiary }\end{array}$ & Pstage \\
\hline 39 & Negative & 7 & 4 & 3 & & T3AN0MX \\
52 & Negative & 6 & 3 & 3 & & T2N0MX \\
54 & Positive & 7 & 4 & 3 & 5 & T3AN0MX \\
54 & Negative & 6 & 3 & 3 & & T2N0MX \\
55 & Negative & 7 & 3 & 4 & & T3BN0MX \\
56 & Negative & 9 & 4 & 5 & 3 & T2N0MX \\
56 & Negative & 7 & 3 & 4 & & T3AN0MX \\
57 & Positive & 6 & 3 & 3 & & T3AN0MX \\
57 & Positive & 7 & 3 & 4 & & T3AN0MX \\
58 & Negative & 7 & 4 & 3 & 5 & T3BN0MX \\
58 & Positive & 7 & 3 & 4 & & T3AN0MX \\
58 & Negative & 7 & 4 & 3 & & T3BN0MX \\
59 & Negative & 7 & 3 & 4 & & T3BN0MX \\
60 & Negative & 7 & 3 & 4 & & T3AN0MX \\
61 & Negative & 7 & 4 & 3 & 5 & T3AN0MX \\
61 & Negative & 7 & 3 & 4 & & T2N0MX \\
62 & Negative & 7 & 3 & 4 & 5 & T3AN0MX \\
62 & Positive & 8 & 4 & 4 & 5 & T3AN0MX \\
63 & Positive & 7 & 3 & 4 & & T3AN0MX \\
65 & Negative & 7 & 3 & 4 & & T2N0MX \\
65 & Negative & 7 & 3 & 4 & 5 & T3AN0MX \\
\hline
\end{tabular}

These are data from the radical prostatectomy specimens. PStage indicates pathological stage (AJCC/UICC, seventh edition) at radical prostatectomy. There were 18 Caucasian and 2 African-American patients.
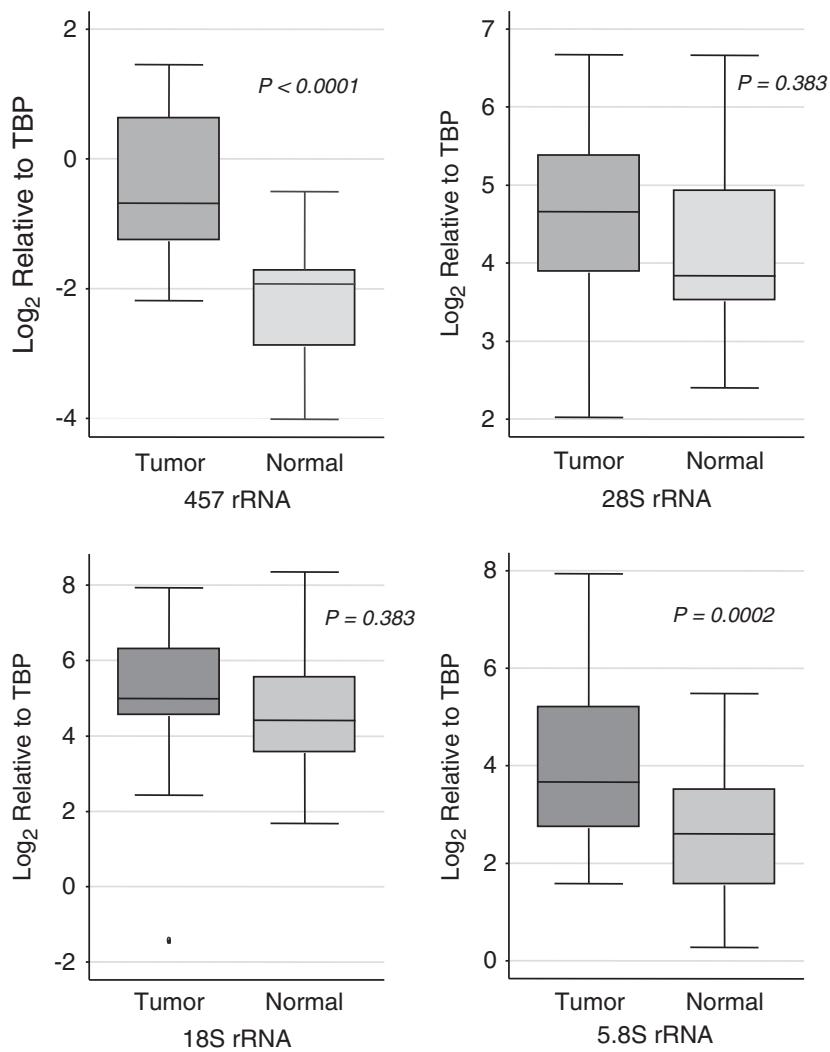

Figure 2 rRNA levels are increased in human prostate cancer tissues. Box plots show the relative levels of the indicated rRNA species in tumor compared with non-tumor (normal) tissue. The sign test was used to test the equality of the matched pairs of observation ( $P$-values are indicated). 
Table 2 Correlations between rRNA species in tumor samples

\begin{tabular}{lcccc}
\hline & $45 S$ & $28 S$ & $18 S$ & $5.8 S$ \\
\hline $45 \mathrm{~S}$ & - & - & - & - \\
$28 \mathrm{~S}$ & $0.4481(0.0417)$ & - & - & - \\
$18 \mathrm{~S}$ & $0.6013(0.0039)$ & $0.5714(0.0068)$ & - & - \\
$5.8 \mathrm{~S}$ & $0.5844(0.0054)$ & $0.5597(0.0083)$ & $0.8584(<0.0001)$ & - \\
\hline
\end{tabular}

Shown are the Spearman's rank correlation coefficients $\rho$ and ( $P$-values) for each pair of variables.

mechanism posited suggests that the extent of $\mathrm{CpG}$ methylation in the rRNA promoter correlates with rRNA levels (Ghoshal et al., 2004). To determine the methylation status of the rDNA promoter we first sought to determine the sequence of the human rDNA promoter region in our cell lines and samples. PCR primers were selected to amplify a $420 \mathrm{bp}$ region that encompassed our bisulfite-sequencing primers and contained additional flanking sequences on either side. The resulting PCR products for each subject were sequenced using the M13 reverse primer. When the sequences of these non-bisulfite-treated genotyping sequences were compared with the published reference sequence for the rRNA promoter region (Genebank accession number: U13369) a number of discrepancies were identified. Interestingly, these sequence variants that we identified were the same as those previously uncovered in a study using human brain DNA (McGowan et al., 2008). Therefore, all the subsequent bisulfite sequencing results of the DNA from cell lines and subjects in this study were compared with this 'corrected' sequence (shown in Figure 1a).

As a control to determine the ability of our bisulfitesequencing approach to detect methylation changes in the rDNA promoter region, we first compared the methylation status in a series of isogenic colo-rectal carcinoma cell lines that contain either wild-type or disrupted versions of DNMT1, DMNT3b or both. A previous study found that targeted disruption of both DNMT1 and DNMT3b resulted in a total lack of methylation in this region (Gagnon-Kugler et al., 2009). Figure 3 shows results of bisulfite sequencing of the rDNA promoter in the colon cancer cell line HCT116 wild-type (HCT116_WT) or knockout mutants that lack either DNMT1 (HCT116_KO1), DNMT3b (HCT116_KO3b) or both genes ( $\left.\overline{\mathrm{H} C T} 116 \_\mathrm{DKO}\right)$. In HCT116_WT, as expected there were two populations of alleles, one densely methylated and the other unmethylated. In HCT116_KO1 cells, there was a reduction in methylation, whereas in HCT116_KO3b cells there was less of a change overall, although the fraction of unmethylated alleles was somewhat increased. In HCT116_DKO cells there was a complete loss of methylation.

To more definitively quantify the extent of methylation of these regions, and to further validate the quantitative nature of our bisulfite sequencing approach, we established a real-time methylation-specific PCR assay for the rDNA promoter. We designed primers, which are complementary to methylated $-158,-9$ and +8 CpGs. It was confirmed by sequencing that PCR products

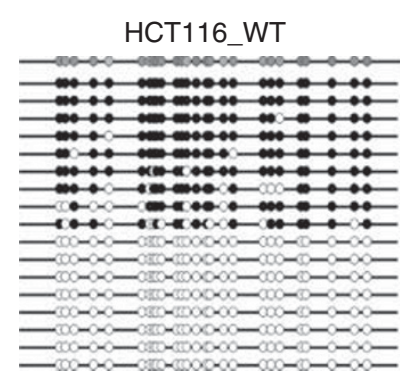

$\mathrm{NIM}=0.41$

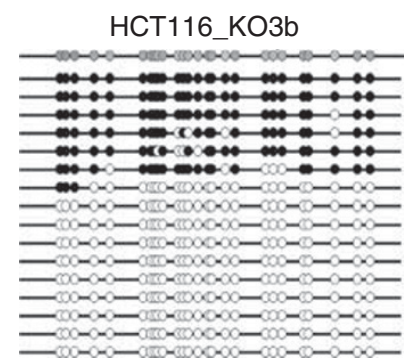

$\mathrm{NIM}=0.33$
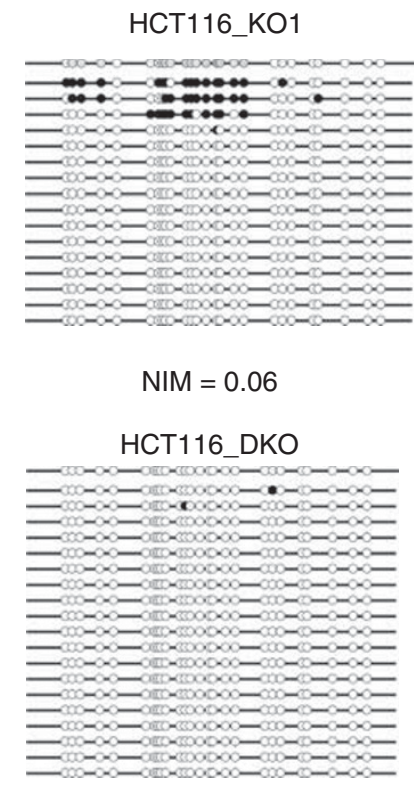

$\mathrm{NIM}=0$

Figure 3 Methylation status of the rRNA promoter in the parental colorectal carcinoma cells HCT116 and its derivative knockout cell lines. The methylation status of each $\mathrm{CpG}$ dinucleotide spanning -158 to +29 bp of human rRNA promoter in HCT116 colorectal carcinoma cells and derivative lines with targeted disruption of DNMT1, DNMT3b or both genes. The rRNA promoter region was amplified from bisulfite-treated genomic DNA and cloned. The 16-19 randomly selected clones from each sample were subjected to automated sequencing. Each row represents the sequence of an individual clone, whereas each column depicts the position of the CpG. The filled and open circles denote methylated and unmethylated $\mathrm{CpGs}$, respectively. The top row shows a heat map of methylated CpGs representing the frequency of methylation in each of the individual clones shown below. The degree of hypermethylation (NIM) at three $\mathrm{CpG}$ islands was assessed using quantitative real-time methylationspecific PCR and was normalized to the amount of bisulfiteconverted input as described in 'Materials and methods.'

obtained using these primer sets were limited to methylated repeats (data not shown). Consistent with the DNA methylation map of HCT116 cells and their mutants, the normalized methylation indices (NIMs) were 0.41 for HCT116_WT, 0.06 for HCT116_KO1, 0.33 for HCT116_KO3B and 0 for HCT116_DKO, respectively (Figure 3 ). Taken together, these findings demonstrate that our bisulfite genomic sequencing approach is reflective of the overall methylation status of the rDNA promoter region.

We next used our bisulfite DNA sequencing approach to determine the methylation status of prostate cancer cell lines and normal prostatic epithelial cells. All of the prostate cancer cell lines and the prostate epithelial cells showed two populations of alleles as seen in the HCT116 wild-type cells (Figure 4). These results indicate that the prostate cancer cell lines tested do not contain hypomethylated alleles in this region, even though they tend to have large, abnormal appearing nucleoli and increased numbers of nucleoli.

We next isolated genomic DNA from the same samples used above for RNA isolation (20 of the 21 


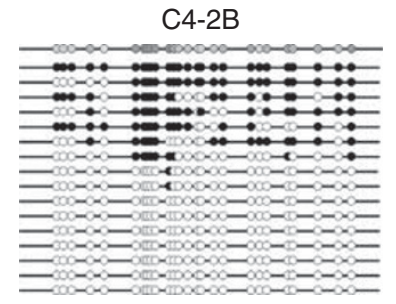

CWR22

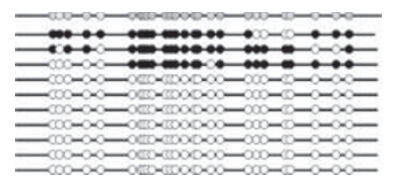

DU145

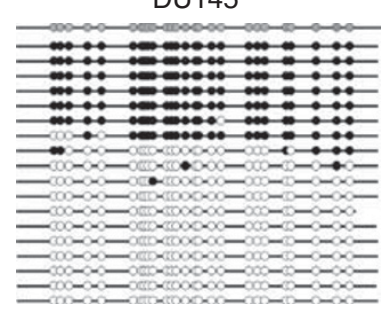

LNCaP

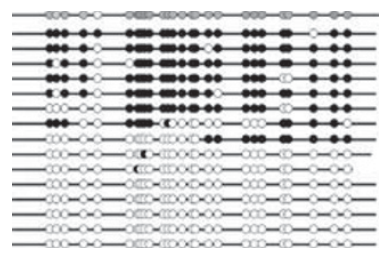

PC-3

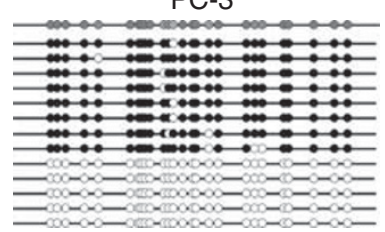

VCaP

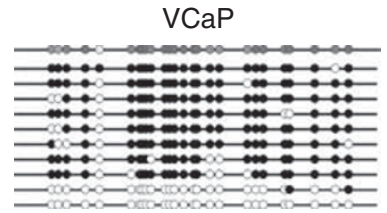

PrEC

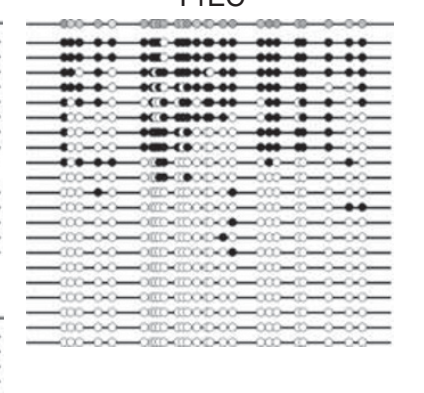

Figure 4 The methylation status of the rRNA promoter in prostate cancer cell lines and normal prostate epithelial cells. The methylation status for each cytosine within each $\mathrm{CpG}$ dinucleotide spanning from -158 to +29 bp of the human rRNA promoter in prostate cancer cell lines and normal prostate epithelial cells was determined. The rRNA promoter region was amplified from bisulfitetreated genomic DNA and cloned. The 10-18 randomly selected clones from each sample were subjected to automated sequencing.

cases contained enough tissue for DNA isolation). Figure 5a shows bisulfite-sequencing results from these human prostate cancer specimens as compared with matched normal specimens. As in the cell lines, all cases showed two populations of alleles. And, there was no clear difference in tumor samples compared with normal samples. A comparison of the methylation pattern of the 20 individual prostates revealed variations among them, which is likely the result of germ line driven polymorphisms in this epigenetic patterning as the differences between individuals were generally present in both tumor and normal tissues (Figure 5b). For example, when comparing tumor with normal samples the fraction of methylated to total $\mathrm{CpGs}$ across the whole region correlated relatively strongly with each other ( $r=0.7663, P=0.0001$, Pearson's moment correlation). It is presently unknown whether this pattern of individual variation of rDNA promoter methylation status in humans is also found in other tissues in the body. When comparing tumor vs normal at individual $\mathrm{CpG}$ sites, there were some relatively subtle differences uncovered such that there was hypomethylation at -30 and -25 in the core promoter region and hypermethylation at +8 in prostate cancer compared with normal tissues (Figure 5c). Despite these minor changes, it is clear that demethylation of rDNA promoter regions does not appear to explain why tumor cell nucleoli are so enlarged, nor why these cells tend to have increased numbers of nucleoli.

We next tested whether the overall fraction of methylated $\mathrm{CpGs}$ in the promoter region correlated with levels of expression of the rRNA species in both tumor and normal. There was no correlation between the fraction of $\mathrm{CpGs}$ methylated in tumor DNA (measured across the whole region) with rRNA levels of any of the rRNA species in the tumor samples suggesting that in human prostate cancer the levels of rRNA transcripts do not correlate with the extent of promoter methylation (not shown). Further, the fraction of methylated $\mathrm{CpGs}$ in the normal tissue did not correlate with any of the rRNA levels in the normal tissues, indicating that the polymorphic nature of the extent of $\mathrm{CpG}$ island methylation of the rDNA promoter does not appear to regulate the levels of rRNA in human prostate tissues (not shown). Finally, the fraction of methylated $\mathrm{CpGs}$ in tumor or normal tissues did not correlate with either Gleason score or pathological stage (not shown). When examining the fraction of methylated individual $\mathrm{CpG}$ sites in tumor or normal tissue, there were no significant correlations between the extent of methylation at these sites and rRNA levels (for any species in tumor or normal) when corrections for multiple testing (Bonferroni) were performed (not shown).

In a recent study we reported that MYC overexpression in human and mouse prostate cancer cell lines was mechanistically related to increased nucleolar size, number and function (Koh et al., 2010). Further, as shown in other tissue types, MYC was shown to positively regulate rRNA levels (Koh et al., 2010). Therefore, we next performed real time reverse transcriptase PCR of MYC mRNA levels on 19 of the same samples used for measuring rRNA levels and there was a significant correlation between the levels of MYC and the 45S (Spearman's rho $=0.5807, P=0.0091$ ), the $18 \mathrm{~S}$ (Spearman's rho $=0.5561, P=0.0134$ ) and the $5.8 \mathrm{~S}$ 
rRNA species (Spearman's rho $=0.4895, P=0.0334$ ), but not with the 28S species (Spearman's $\rho=0.3140$, $P=0.1904)$.
As rRNA levels were increased in the human prostate cancer samples, it was of interest to determine whether these levels related to nucleolar size and number. a

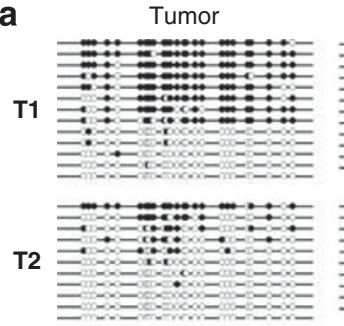

T3

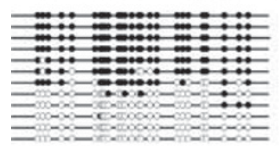

T4

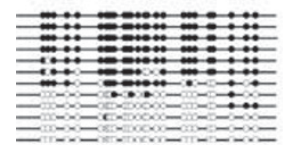

T5
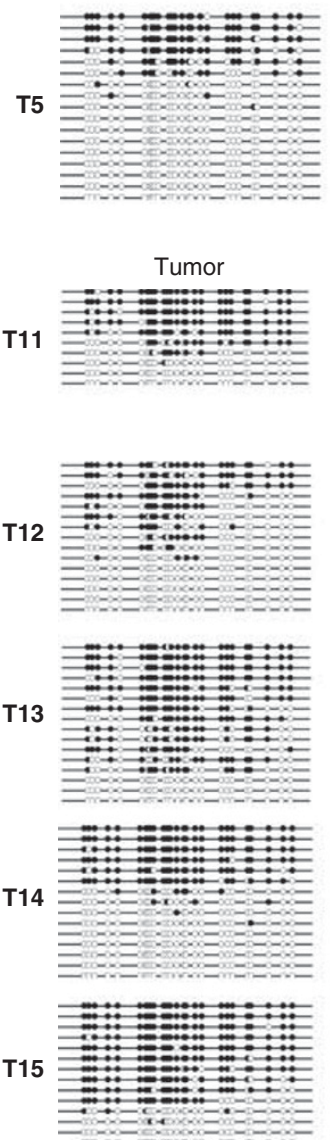
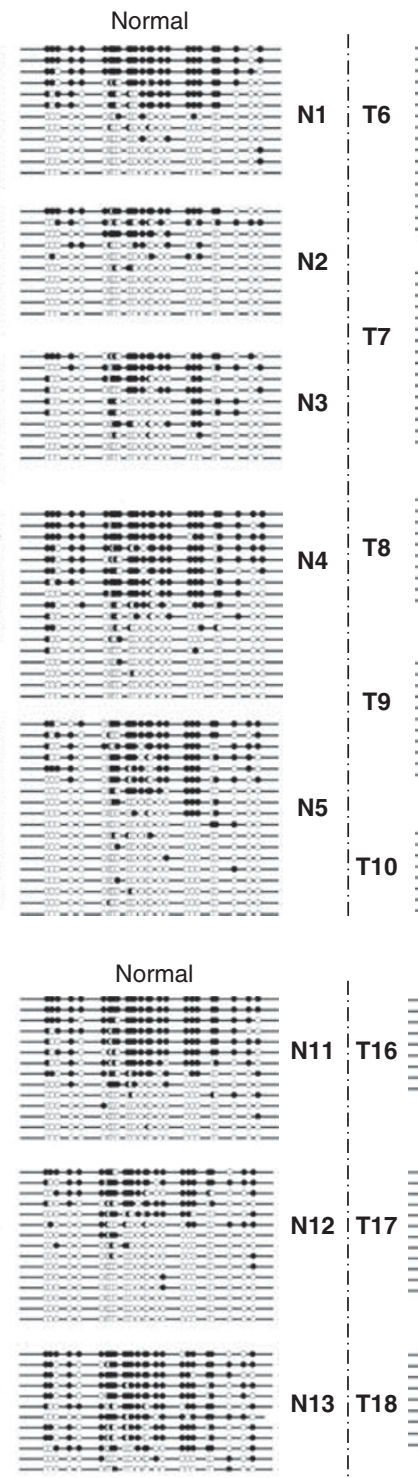

Tumor
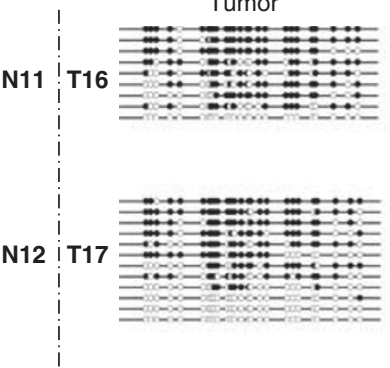

N13 ${ }^{i}{ }^{T} 18$
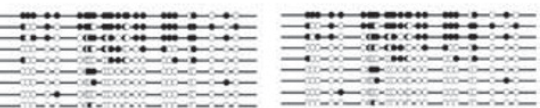

N18
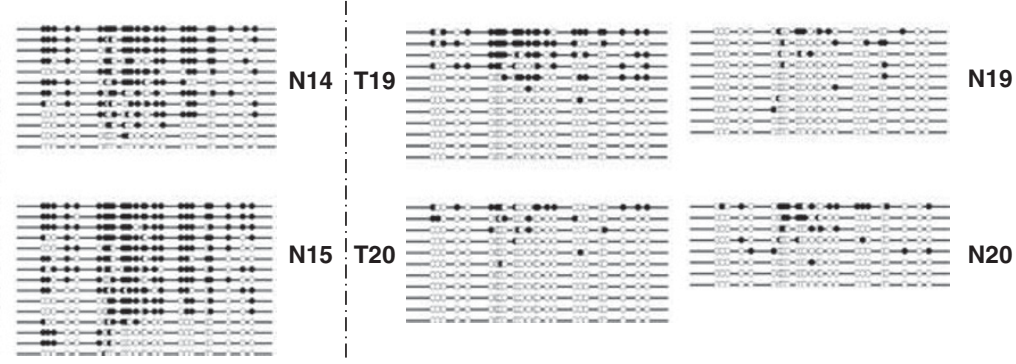

Figure 5 The methylation status of the rRNA promoter in clinical prostate cancer relative to matched normal tissues. (a) The methylation status of each cytosine within each $\mathrm{CpG}$ dinucleotide spanning -158 to $+29 \mathrm{bp}$ of the human rRNA promoter in 20 human prostate cancers and matched normal tissues. The rRNA promoter region was amplified from bisulfite-treated genomic DNA and cloned. The 10-18 randomly selected clones from each sample were subjected to automated sequencing. (b) Quantitative analysis of methylation density at each $\mathrm{CpG}$ and all $\mathrm{CpGs}$ with respect to the +1 site of the rRNA promoter in individual tumors and matched normal prostate tissues is shown. The percentage of clones methylated and unmethylated at each position among clones is represented in this bar diagram. $\mathrm{T}$ and $\mathrm{N}$ denote prostate cancer and normal tissue, whereas Unmet and Met indicate unmethylated and methylated $\mathrm{CpGs}$, respectively. The average percentage of methylation for all $\mathrm{CpG}$ sites at each $\mathrm{CpG}$ site, for prostate cancer $(N=20$; left-side bars) and matched normal tissues $\left(N=20\right.$; right-side bars). Data are expressed as $* P<0.05$; ** $P<0.01$, measured by $\chi^{2}$-test. 


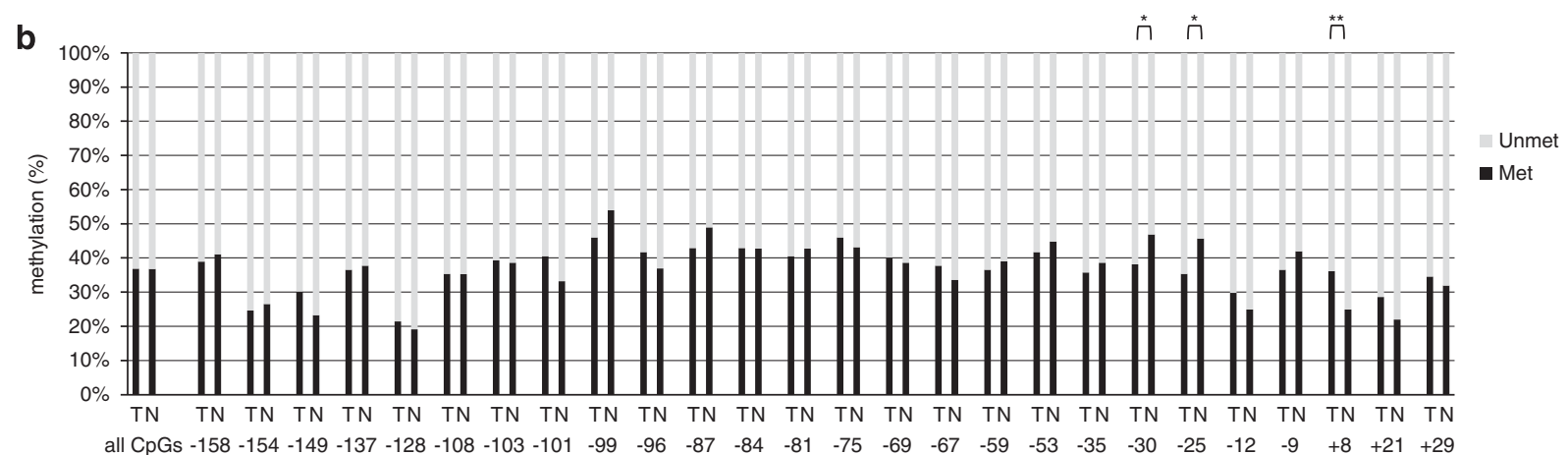

Figure 5 Continued.

Therefore, we performed immunohistochemical staining for fibrillarin, a well-known marker of nucleoli, which we recently reported was directly regulated by MYC and levels of fibrillarin correlated with MYC protein levels in clinical samples (Koh et al., 2010). Although levels of fibrillarin protein did correlate with levels of MYC mRNA in tumor samples (Spearman's rho $=0.5702$, $P=0.0108$ ), fibrillarin protein levels in tumor samples did not correlate with rRNA levels for any of the species in the tumor samples, albeit levels of fibrillarin in the non-neoplastic tissues did correlate with levels of the $18 \mathrm{~S}$ (Spearman's rho $=0.5421, P=0.0165$ ) and $5.8 \mathrm{~S}$ (Spearman's rho $=0.4947, P=0.0313$ ) species in the non-neoplastic tissues from these same cases.

\section{Discussion}

Although it is generally assumed that rRNA levels are elevated in human cancers, very few clinical samples have ever been investigated in this regard (Williamson et al., 2006). In this manuscript we show for the first time that levels of rRNA are elevated in human prostate cancer clinical specimens as compared with matched non-neoplastic tissues. What is the mechanism for such regulation? The current model for transcriptional regulation of ribosomal DNA (rDNA), which is composed of hundreds of copies of rRNA genes, proposes two main mechanisms. For short-term regulation, the transcription rate at euchromatic active rDNA is altered by reversible modification of Pol I transcription factors that affect the efficiency of transcription initiation and/or the rate of transcription (McStay and Grummt, 2008; Drygin et al., 2010). Although a number of pathways that are altered in prostate cancer could potentially affect nucleolar dynamics (Fischer et al., 2004), until recently very little was known regarding which if any of these pathways do affect prostate cancer nucleoli. Others have previously shown that MYC can activate rRNA transcription and we have recently shown that MYC does regulate levels of $45 \mathrm{~S}, 28 \mathrm{~S}, 18 \mathrm{~S}$ and $5.8 \mathrm{~S}$ rRNA in human prostate cancer cells (Koh et al., 2010) (and M Uemura and AM De Marzo, unpublished observations). In fact, it was shown that MYC overexpression in both mouse and human prostate cells results in increased nucleolar size, number, as well as an increase in a nucleolar program of gene expression including fibrillarin, nucleolin and UBTF (Koh et al., 2010). Taken together with previous studies that have shown that MYC can regulate nucleolar genes and nucleolar architecture in other cell types (Schlosser et al., 2003; Grandori et al., 2005; Grewal et al., 2005), and the present findings that MYC mRNA levels correlated with rRNA levels and fibrillarin protein in the present samples, these results suggest that MYC overexpression can provide a molecular mechanism that may explain, at least in part, why nucleoli are altered in prostatic intraepithelial neoplasia and prostatic adenocarcinoma. Whether other molecular alterations that affect the epigenetic state of the rDNA loci also affect nucleolar dynamics in prostate cancer, and precisely how MYC can regulate nucleolar size, number and function awaits additional studies. Interestingly, recent findings are beginning to elucidate how MYC affects rRNA transcription by both inducing gene loop structures in rDNA chromatin that juxtaposes upstream and downstream rDNA sequences, as well as, by inducing other changes in chromatin structure including histone modifications (Shiue et al., 2009, 2010).

By contrast to this short-term regulation, in some cases long-term regulation during development and differentiation is mediated by epigenetic mechanisms that consist of recruitment of previously inactive heterochromatic repeats to become active repeats (McStay and Grummt, 2008). In the latter case, specific chromatin modifications alter the ratio of active to silent copies of rRNA genes, thereby adjusting the number of genes that are involved in transcription (Drygin et al., 2010). Thus, it is reasonable to hypothesize that the increased rRNA levels and nucleolar size, number and function in cancer may relate to recruitment of inactive repeat units to become active repeats. One previous study examined this question and reported that in hepatocellular carcinoma there was an apparent hypomethylation of the rDNA promoter region that the authors indicated could account for increased rRNA levels (Ghoshal et al., 2004). In the present study in human prostate cancer, we found that the DNA methylation status of rDNA promoter region is essentially unchanged in tumor samples as compared 
with matched normal samples. Thus, such changes cannot explain differences in rRNA levels or why there are nucleolar structural abnormalities in prostate cancer. At present we cannot explain the apparent discrepancy found in terms of methylation state of the rDNA locus in prostate cancer as compared with hepatocellular cancer. Additional studies of the $\mathrm{CpG}$ methylation status of these loci in both of these cancers and other cancers will be needed to ultimately clarify this issue.

Although all rRNA species were upregulated in prostate tumors as compared with matched nonneoplastic tissues, the overexpression reached statistical significance only for the $45 \mathrm{~S}$ and $5.8 \mathrm{~S}$ species. At this time we do not have a molecular explanation for this apparent discrepancy. In terms of the $45 \mathrm{~S}$ species, which is considered to be a sign of new transcription, our results support the hypothesis that the rate of rRNA synthesis at this locus is increased in tumor samples. In terms of the other rRNA species examined, it is well known that the processing of the $45 \mathrm{~S}$ transcript into the mature $28 \mathrm{~S}, 18 \mathrm{~S}$ and $5.8 \mathrm{~S}$ species is a multistep process that is subject to a number of potential modes of regulation. For example, IGF-1 signaling primarily effects 18S rRNA processing and thus the function in the $40 \mathrm{~S}$ ribosomal subunit, with less effects on the $28 \mathrm{~S}$ and 5.8S levels (McMahon et al., 2010). Thus, we expect that a number of factors regulate steady state levels of rRNA species in prostate cancer and that the regulation of each may be somewhat decoupled from each other. Nevertheless, the levels of all of the different species in a given patient did correlate fairly strongly with each other. As a result, it is perhaps more likely that the reason that not all species reached statistical significance (in terms of overexpression in the cancer tissues) was the result of the relatively small number of samples that were assayed in this study.

In summary, we found that rRNA levels are markedly elevated in human clinical prostate cancer cases, but there was no evidence, using both prostate cancer cell lines and a range of clinical specimens, to indicate that rDNA promoter hypomethylation could be responsible for either this overexpression or for altered nucleolar structure and function in prostate cancer.

\section{Materials and methods}

\section{Cell culture}

LNCaP, PC-3, 22Rv1 and DU 145 cell lines were obtained from American Type Culture Collection (Rockville, MD, USA). Prostate epithelial cells were obtained from Lonza (Basel, Switzerland). C4-2B and VCaP cell lines were generously provided by Dr John T Isaacs (Johns Hopkins University, Baltimore, MD, USA). Prostate cell lines were propagated in RPMI 1640 (Invitrogen, Carlsbad, CA, USA) containing 10\% fetal bovine serum (Hyclone, Waltham, MA, USA). The HCT116 colorectal carcinoma cell line (HCT116 WT) and the DNMT1 ${ }^{-} /^{-}\left(\mathrm{HCT} 116 \_\mathrm{KO} 1\right), \mathrm{DNMT}^{-} \mathrm{b}^{-}{ }^{-}$ (HCT116_KO3b), and DNMT1 ${ }^{-} /-$, DNMT3b ${ }^{-} /^{-}$(HCT116 $\mathrm{DKO})$ cell lines were generously provided by B Vogelstein and were grown as previously described (Rhee et al., 2002). All cells were grown in $5 \% \mathrm{CO} 2 / 95 \%$ air at $37^{\circ} \mathrm{C}$.
Clinical tissue samples

This study was conducted with the approval of the Johns Hopkins Medical Institutions Institutional Review Board. Primary prostate cancers were obtained from 21 men undergoing radical prostatectomy at the Johns Hopkins Hospital for clinically localized prostate cancer (see Table 1). All tissue samples were obtained by sectioning the prostates immediately after surgery and identifying regions of cancerous and noncancerous tissue that were verified by frozen section analysis. All tumor tissues were estimated to be $\sim 70-80 \%$ tumor cells, and none of the non-cancerous tissues contained histological evidence of carcinoma. None of these patients had received androgen deprivation therapy. These tissues were snap frozen and stored at $-80{ }^{\circ} \mathrm{C}$. Gleason score, pathological stage and patient age were collected for each of the subjects at the time of radical prostatectomy (Table 1$)$.

\section{RNA extraction}

RNA was extracted from OCT-embedded frozen tissues using Trizol (Invitrogen) following the manufacturer's instructions. Briefly $5 \mu \mathrm{m}$ frozen tissue sections $(n=50)$ were treated with Trizol to obtain a clear lysate solution. After chloroform extraction, total RNA was recovered from the aqueous phase by precipitation with isopropyl alcohol. The pellet was washed by $75 \%$ ethanol and dissolved in diethylpyrocarbonate-treated water. Total RNA was treated with DNAse I (Biolabs, cat\# $\mathrm{M} 0303 \mathrm{~s}$ ) at $37^{\circ} \mathrm{C}$ for $20 \mathrm{~min}$ to remove trace genomic DNA. Total RNA was quantified spectrophotometrically and stored as aliquots at $-80{ }^{\circ} \mathrm{C}$.

\section{Complementary (c)DNA synthesis}

Reverse transcription of RNA was performed using the BioRad iScript cDNA synthesis kit (Cat. No. 1780-8891) (Bio$\mathrm{Rad})$ in a final volume of $20 \mu \mathrm{l}$ containing $1 \times$ iScript reaction mix, $1 \mu$ RNAse $\mathrm{H}+$ reverse transcriptase, $200 \mathrm{ng}$ total RNA and nuclease-free water. The samples were incubated at $25^{\circ} \mathrm{C}$ for $5 \mathrm{~min}$ and $42^{\circ} \mathrm{C}$ for $30 \mathrm{~min}$, and reverse transcriptase was inactivated by heating at $85^{\circ} \mathrm{C}$ for $5 \mathrm{~min}$.

\section{Reverse transcriptase PCR amplification}

All PCR reactions were performed using Bio-Rad MyiQ single color Real-Time PCR detection system. For each PCR run (except for the 45S rRNA precursor), a master mix was prepared on ice with $1 \times$ PCR buffer, $200 \mu \mathrm{m}$ dUTP, $1.5 \mathrm{~mm}$ $\mathrm{MgCl}_{2}, 200 \mathrm{nM}$ forward and reverse primer, 0.028 units of Ampli Taq Gold DNA polymerase (Roche) and 1:100000 SYBR Green (Molecular Probes). A volume of $1 \mu \mathrm{l}$ of appropriate diluted cDNA sample was added to $25 \mu \mathrm{l}$ of the PCR master mix. The thermal cycling conditions comprised an initial denature step at $95^{\circ} \mathrm{C}$ for $5 \mathrm{~min}, 45$ cycles at $95^{\circ} \mathrm{C}$ for $30 \mathrm{~s}, 60^{\circ} \mathrm{C}$ for $30 \mathrm{~s}$ and $72^{\circ} \mathrm{C}$ for $30 \mathrm{~s}$. For the $45 \mathrm{~S}$ rRNA precursor we used GreenER qPCR SuperMix (Bio-Rad) instead of the master mix listed above. Otherwise, the thermal cycling conditions were the same as above.

Relative copy numbers for each target rDNA gene, MYC and reference gene $(T B P)$ were determined using the relative standard curve method (Applied Biosystems User Bulletin \#2) (Applied Biosystems, Foster, CA, USA), with the standard curve (in triplicate reactions) comprised of serially diluted cDNA generated after total RNA isolation from PC3 cells. Each cDNA sample was run in six replicate wells and appropriate dilute RNAs for each sample were used for negative controls. rRNA species abundance was normalized to the abundance of TBP transcripts for each tumor and normal specimen. All primer sequences are shown in Table 3. 
Table 3 Primers used in this study

\begin{tabular}{|c|c|c|c|}
\hline Primer & Position & Sequence & Product Size ( $b p)$ \\
\hline 45S rRNA forward & $851-868$ & 5'-GAACGGTGGTGTGTCGTT-3' & 130 \\
\hline 45S rRNA reverse & $961-980$ & 5'-GCGTCTCGTCTCGTCTCACT-3' & \\
\hline 28S rRNA forward & $8352-8371$ & 5'-AGAGGTAAACGGGTGGGGTC-3' & 113 \\
\hline 28S rRNA reverse & $8448-8464$ & 5'-GGGGTCGGGAGGAACGG-3' & \\
\hline 18S rRNA forward & $4026-4043$ & $5^{\prime}$-GATGGTAGTCGCCGTGCC- $3^{\prime}$ & 110 \\
\hline 18S rRNA reverse & $4119-4135$ & 5'-GCCTGCTGCCTTCCTTGG-3' & \\
\hline 5.8S rRNA forward & $6642-6657$ & $5^{\prime}$-ACTCGGCTCGTGCGTC-3' & 136 \\
\hline 5.8S rRNA reverse & $6762-6777$ & $5^{\prime}$-GCGACGCTCAGACAGG-3' & \\
\hline TBP forward & & 5'-CACGAACCACGGCACTGATT-3' & 89 \\
\hline TBP reverse & & 5'-TTTTCTTGCTGCCAGTCTGGAC-3' & \\
\hline rDNA genotyping forward & & 5'-GTGTGTCCCGGTCGTAGG-3' & 420 \\
\hline rDNA genotyping reverse & & 5'-GTCACCGTGAGGCCAGAG-3' & \\
\hline rDNA promoter bisulfite sequence foward & & 5'-GTTTTTGGGTTGATTAGA-3' & 229 \\
\hline rDNA promoter bisulfite sequence reverse & & 5'-AAAACCCAACCTCTCC-3' & \\
\hline rDNA promoter RTMSP forward & & 5'-GGGGTTGATTAGAGGGTTTC-3' & 189 \\
\hline rDNA promoter RTMSP reverse & & 5'-CAACGTATCAACAATAACCCG A-3' & \\
\hline MYOD1 RTMSP forward & & 5'-ССААСТССАААТССССТСТСТАТ- $3^{\prime}$ & 162 \\
\hline MYOD1 RTMSP reverse & & 5'-TGATTAATTTAGATTGGGTTTAGAGAAGGA-3' & \\
\hline mMYC forward & & 5'-CAGATCAGCAACAACCGAAA-3' & 168 \\
\hline mMYC reverse & & 5'-GGCCTTTTCATTGTTTTCCA-3' & \\
\hline
\end{tabular}

\section{DNA extraction and bisulfite treatment}

Genomic DNA was extracted from frozen matched pairs of tumor and normal tissues obtained from the same radical prostatectomy specimens, as well as from cancer cell lines and non-neoplastic prostatic prostate epithelial cells using the DNeasy Kit (QIAGEN, Valencia, CA, USA) and following the manufacturer's protocol. In all $500 \mathrm{ng}$ of genomic DNA from cell lines and $10 \mathrm{ng}$ of DNA from clinical tissues were treated with sodium bisulfite in order to convert cytosine residues to uracil using the EZ Methylation Gold-Kit (Zymo Research, Orange, CA, USA). The converted DNA was eluted in $40 \mu \mathrm{l}$ of distilled water.

\section{PCR, genotyping and bisulfite genomic sequencing}

For initial PCR genotyping of the rDNA locus, $1 \mu 1$ of human male genomic DNA (EMD Chemicals, Gibbstown, NJ, USA) was amplified by PCR using platinum Taq DNA polymerase (Invitrogen). For bisulfite DNA sequencing, $\sim 1 \mu 1$ of the post bisulfite-treated product was amplified by PCR using platinum Taq DNA polymerase (Invitrogen). Each PCR reaction consisted of a $95^{\circ} \mathrm{C}, 4 \mathrm{~min}$ initial denaturation and enzyme activation step followed by 35 cycles at $98^{\circ} \mathrm{C}$ for $30 \mathrm{~s}$, $50{ }^{\circ} \mathrm{C}$ for $45 \mathrm{~s}$ (for genotyping PCR, $55^{\circ} \mathrm{C}$ for $45 \mathrm{~s}$ ) and $72{ }^{\circ} \mathrm{C}$ for $45 \mathrm{~s}$, on the i-Cycler IQ (Bio-Rad Laboratories, Hercules, CA, USA). Bisulfite conversion of all the non-methylated deoxycytosines resulted in the creation of uracil residues that were later transformed during the PCR amplification to thymine. Because the primers did not contain $\mathrm{CpG}$ dinucleotides, methylated and unmethylated sequences amplified with equal efficiency. The resulting product was excised, purified, subcloned and transformed (pCR2.1-TOPO TA cloning kit, Invitrogen). Plasmid DNA from at least eight positive clones from the bisulfite genomic sequencing product was sequenced using an ABI 3730 DNA Analyzer (Applied Biosystems). To ensure that the bisulfite conversion was complete, only clones in which all cytosine residues in non$\mathrm{CpG}$ dinucleotides had been converted to thymine were included in the analysis.

\section{Real time methylation-specific PCR}

RT-methylation-specific PCR was carried out using a previously described methodology (Yegnasubramanian et al., 2004). Briefly, sodium bisulfite treatment of DNA will result in conversion of deoxycytidine residues to deoxyuridine unless the deoxycytidines are methylated at the $5^{\prime}$ position. To ascertain the completeness of the conversion reaction within the input templates in each sample, bisulfite-treated DNA was amplified using real-time PCR with oligonucleotide primers complementary to a region of the MYOD1 promoter that did not contain any $\mathrm{CpG}$ dinucleotides but did contain non-CpG cytosines. Hypermethylation was then examined by real-time PCR amplification of bisulfite-modified DNA using oligonucleotide primers specific to a fully methylated bisulfiteconverted portion of each $\mathrm{CpG}$ island such that only $\mathrm{CpG}$ islands that were methylated at every $\mathrm{CpG}$ dinucleotide interrogated by the primers would be amplified and generate a fluorescent signal. All PCR reactions were carried out using an i-Cycler IQ at $95^{\circ} \mathrm{C}$ for 3 min followed by 40 cycles of $95^{\circ} \mathrm{C}$ for $30 \mathrm{~s}, 54^{\circ} \mathrm{C}$ for $30 \mathrm{~s}$ and $72{ }^{\circ} \mathrm{C}$ for $45 \mathrm{~s}$. The MYOD1 reaction was carried out under the same conditions except that an annealing temperature of $60^{\circ} \mathrm{C}$ was used. Each PCR reaction was carried out in a $20 \mu \mathrm{l}$ volume containing $10 \mu \mathrm{l}$ iQSYBRGreen (Bio-Rad Laboratories), $1 \mu \mathrm{M}$ forward primer, $1 \mu \mathrm{M}$ reverse primer, $1 \mu \mathrm{l}$ of bisulfite-modified DNA. The SssI methylase is known to methylate deoxycidine residues within $\mathrm{CpG}$ dinucleotides, and this reaction can be driven to completion. Thus, SssI treatment was used to generate a fully methylated DNA template in control DNA samples obtained from donor WBCs as a positive control and was used to generate a standard curve to quantify the amount of fully methylated promoter sequences in each reaction. The normalized index of methylation (NIM) was defined as the ratio of the normalized amount of methylated templates at the promoter of interest to the normalized amount of converted MYOD1 templates in any given sample. That is,

$$
\begin{aligned}
\mathrm{NIM}= & {[(\text { GENEsample }) /(\text { GENESssI })] / } \\
& {[(\text { MYODIsample }) /(\text { MYODSss })] }
\end{aligned}
$$

where (GENEsample) is the number of fully methylated copies of the gene of interest in a given sample, (GENESssI) is the number of fully methylated copies of the gene of interest in the SssI universally methylated control DNA, (MYOD1sample) is the number of MYOD1 copies in a given sample, and (MYOD1SssI) is the number of MYOD1 copies in the SssI universally methylated DNA. The NIM serves as an index of 
the percentage of bisulfite converted input copies of DNA that are fully methylated at the primer hybridization sites. Therefore, in diploid samples that are fully methylated at the region of interest the maximum NIM is 1 , and any fraction less than 1 indicates that less than $100 \%$ of the templates were methylated. In samples in which the template DNA is completely unmethylated at the primer sites, the NIM would be 0 . It is important to note that the NIM may be $>1$ if copies of MYOD1 are deleted relative to the gene of interest, or copies of the gene of interest are gained relative to MYOD1 in any given sample.

\section{Immunohistochemical staining}

Immunohistochemical staining and quantitative image analysis of frozen prostate tissue sections from the same specimens used for RNA isolation and DNA isolation were performed as described (Koh et al., 2010).

\section{References}

Bostwick DG, Pacelli A, Lopez-Beltran A. (1996). Molecular biology of prostatic intraepithelial neoplasia. Prostate 29: 117-134.

Derenzini M, Trere D, Pession A, Montanaro L, Sirri V, Ochs RL. (1998). Nucleolar function and size in cancer cells. Am J Pathol 152: 1291-1297.

Drygin D, Rice WG, Grummt I. (2010). The RNA polymerase I transcription machinery: an emerging target for the treatment of cancer. Annu Rev Pharmacol Toxicol 50: 131-156.

Epstein JI, Yang XJ. (2002). Prostate Biopsy Interpretation, 3rd edn. Lippincott Williams \& Wilkins: Philadelphia.

Espada J, Ballestar E, Santoro R, Fraga MF, Villar-Garea A, Nemeth A et al. (2007). Epigenetic disruption of ribosomal RNA genes and nucleolar architecture in DNA methyltransferase 1 (Dnmt1) deficient cells. Nucleic acids res 35: 2191-2198.

Fischer AH, Bardarov Jr S, Jiang Z. (2004). Molecular aspects of diagnostic nucleolar and nuclear envelope changes in prostate cancer. J Cell Biochem 91: 170-184.

Gagnon-Kugler T, Langlois F, Stefanovsky V, Lessard F, Moss T. (2009). Loss of human ribosomal gene CpG methylation enhances cryptic RNA polymerase II transcription and disrupts ribosomal RNA processing. Mol Cell 35: 414-425.

Ghoshal K, Majumder S, Datta J, Motiwala T, Bai S, Sharma SM et al. (2004). Role of human ribosomal RNA (rRNA) promoter methylation and of methyl-CpG-binding protein MBD2 in the suppression of rRNA gene expression. $J$ Biol Chem 279: 6783-6793.

Gonzalez IL, Sylvester JE. (1995). Complete sequence of the 43-kb human ribosomal DNA repeat: analysis of the intergenic spacer. Genomics 27: 320-328.

Grandori C, Gomez-Roman N, Felton-Edkins ZA, Ngouenet C, Galloway DA, Eisenman RN et al. (2005). c-Myc binds to human ribosomal DNA and stimulates transcription of rRNA genes by RNA polymerase I. Nat cell biol 7: 311-318.

Grewal SS, Li L, Orian A, Eisenman RN, Edgar BA. (2005). Myc-dependent regulation of ribosomal RNA synthesis during Drosophila development. Nat cell biol 7: 295-302.

Grummt I. (2010). Wisely chosen paths - regulation of rRNA synthesis: delivered on 30 June 2010 at the 35th FEBS Congress in Gothenburg, Sweden. FEBS J 277: 4626-4639.

Grummt I, Voit R. (2010). Linking rDNA transcription to the cellular energy supply. Cell cycle (Georgetown, Tex) 9: 225-226.

Haltiner MM, Smale ST, Tjian R. (1986). Two distinct promoter elements in the human rRNA gene identified by linker scanning mutagenesis. Mol Cell Biol 6: 227-235.
Statistical analyses

All statistical analyses were performed using Stata 8.0 data analysis and statistical software for Microsoft Windows.

\section{Conflict of interest}

The authors declare no conflict of interest.

\section{Acknowledgements}

This study was supported by the Patrick C Walsh Prostate Cancer Fund of which AMD is the Peter J Sharp Scholar, and Prostate SPORE P50CA58236. We wish to thank Helen Fedor and the Brady Urological Institute Prostate Specimen Repository for fresh prostate tissues.

Koh C, Gurel B, Sutcliffe S, Aryee M, Uemura M, Zeller K et al (2010). Fibrillarin links MYC to nucleolar function and stem cell renewal in prostate cancer. Am J Pathol 4: 1824-1834.

McGowan PO, Sasaki A, Huang TC, Unterberger A, Suderman M, Ernst C et al. (2008). Promoter-wide hypermethylation of the ribosomal RNA gene promoter in the suicide brain. PloS one 3 : e2085.

McMahon M, Ayllon V, Panov KI, O'Connor R. (2010). Ribosomal $18 \mathrm{~S}$ RNA processing by the IGF-I-responsive WDR3 protein is integrated with $\mathrm{p} 53$ function in cancer cell proliferation. $J$ Biol Chem 285: $18309-18318$.

McStay B, Grummt I. (2008). The epigenetics of rRNA genes: from molecular to chromosome biology. Annu Rev Cell Dev Biol 24: 131-157.

Rhee I, Bachman KE, Park BH, Jair KW, Yen RW, Schuebel KE et al. (2002). DNMT1 and DNMT3b cooperate to silence genes in human cancer cells. Nature 416: 552-556.

Ruggero D, Pandolfi PP. (2003). Does the ribosome translate cancer? Nat Rev Cancer 3: 179-192.

Schlosser I, Holzel M, Murnseer M, Burtscher H, Weidle UH, Eick D. (2003). A role for c-Myc in the regulation of ribosomal RNA processing. Nucleic acids res 31: 6148-6156.

Shiue CN, Berkson RG, Wright AP. (2009). c-Myc induces changes in higher order rDNA structure on stimulation of quiescent cells. Oncogene 28: 1833-1842.

Shiue CN, Arabi A, Wright AP. (2010). Nucleolar organization, growth control and cancer. Epigenetics 5: 200-205.

White RJ. (2005). RNA polymerases I and III, growth control and cancer. Nat Rev Mol Cell Biol 6: 69-78.

Williamson D, Lu YJ, Fang C, Pritchard-Jones K, Shipley J. (2006). Nascent pre-rRNA overexpression correlates with an adverse prognosis in alveolar rhabdomyosarcoma. Genes Chromosomes Cancer 45: 839-845.

Yegnasubramanian S, Kowalski J, Gonzalgo ML, Zahurak M, Piantadosi S, Walsh PC et al. (2004). Hypermethylation of $\mathrm{CpG}$ islands in primary and metastatic human prostate cancer. Cancer Res 64: 1975-1986.

This work is licensed under the Creative Commons Attribution-NonCommercial-No Derivative Works 3.0 Unported License. To view a copy of this license, visit http://creativecommons.org/licenses/by-nc-nd/3.0/ 